\title{
Effects of multiple electronic shells on strong-field multiphoton ionization and high-order harmonic generation of diatomic molecules with arbitrary orientation: An all-electron time-dependent density-functional approach
}

\author{
Dmitry A. Telnov ${ }^{1,2, *}$ and Shih-I Chu (朱時宜) $)^{2, \dagger}$ \\ ${ }^{1}$ Department of Physics, St. Petersburg State University, 198504 St. Petersburg, Russia \\ ${ }^{2}$ Department of Chemistry, University of Kansas, Lawrence, Kansas 66045, USA
}

(Received 27 July 2009; published 20 October 2009)

\begin{abstract}
We present a time-dependent density-functional theory approach with proper long-range potential for an $a b$ initio study of the effect of correlated multielectron responses on the multiphoton ionization (MPI) and highorder harmonic generation (HHG) of diatomic molecules $\mathrm{N}_{2}$ and $\mathrm{F}_{2}$ in intense short laser pulse fields with arbitrary molecular orientation. We show that the contributions of inner molecular orbitals to the total MPI probability can be sufficiently large or even dominant over the highest-occupied molecular orbital, depending on detailed electronic structure and symmetry, laser field intensity, and orientation angle. The multielectron effects in HHG are also very important. They are responsible for enhanced HHG at some orientations of the molecular axis. Even strongly bound electrons may have a significant influence on the HHG process.
\end{abstract}

DOI: $10.1103 /$ PhysRevA.80.043412

PACS number(s): 33.80.Rv, 42.65.Ky, 32.80.Rm

\section{INTRODUCTION}

Recent advances in laser technology have made possible experimental investigation of atomic and molecular processes on an ultrashort attosecond time scale [1]. High intensities which can be reached at short times allow probing valence and core electrons and measuring the atomic and molecular structure [2]. Due to the extra internuclear degrees of freedom, the responses of molecules to strong fields are considerably more complicated than that of atoms and pose additional challenge to researchers. The simplest diatomic molecules subject to intense laser fields continue attracting much attention and have been a subject of many experimental and theoretical investigations [3]. Revolutionary improvements of experimental techniques have made possible impressive observations and measurements on diatomic molecules including molecular axis orientation effects, multiple molecular orbitals (MO) contributions, electron diffraction, MO imaging etc. [4-9] (see also the review paper [10] and references therein). Multiphoton ionization (MPI) is one of the fundamental atomic and molecular processes that take place in strong laser fields. Recent experiments with diatomic molecules $[6,7]$ were able to perform direct measurements of dependence of the ionization signal on the orientation of the molecular axis with respect to the polarization of the laser field. Thus the theoretical description of the orientation dependence of MPI remains an important and timely task. Another important and actively studied process is the high-order harmonic generation (HHG) which can serve as a source of coherent extreme ultraviolet radiation with many applications in the attosecond physics [1,2].

Traditionally many theoretical studies of MPI and HHG processes in molecules are based on the models \{molecular Keldysh-Faisal-Reiss (KFR) model [11] and molecular Ammosov-Delone-Krainov (ADK) [12] model\} which can

\footnotetext{
*telnov@pcqnt1.phys.spbu.ru

†sichu@ku.edu
}

be regarded as various implementations of the strong-field approximation (SFA). While these models result in rather simple theoretical expressions and are capable of providing some qualitative predictions, they can fail to explain some experimental observations in stronger fields. Although many attempts have been made recently to improve SFA (see, e.g., [13-15] and references therein), it has intrinsic restrictions and cannot compete with $a b$ initio calculations for accuracy of the results. Besides other limitations, SFA-based theories usually deal only with the highest-occupied molecular orbital (HOMO) and neglect the multielectron dynamics of the target molecules. However, multielectron effects due to the electron exchange and correlation may be significant even when the inner electrons are strongly bound and are not excited by the driving laser field $[16,17]$.

In the recent Rapid Communication [18], we extended the time-dependent density-functional theory (TDDFT) with proper long-range potential $[19,20]$ to an all-electron threedimensional (3D) ab initio study of the MPI of diatomic molecules $\mathrm{N}_{2}, \mathrm{~F}_{2}$, and $\mathrm{O}_{2}$ with arbitrary molecular axis orientation, a subject of much current experimental interests [6-9]. Our calculations show that the contributions of the inner-shell MO to the MPI signal can be very significant and even dominant over the HOMO contribution, depending upon the target molecule, orientation angle, and the intensity of the laser field, in general good agreement with the most recent experimental results $[6,7]$. Very recently, a similar TDDFT approach was successfully applied to the MPI study of the $\mathrm{CO}_{2}$ molecule and also revealed an importance of multiple orbital contributions [21].

In this paper, we extend the approach of Ref. [18] to further investigation of the molecules $\mathrm{N}_{2}$ and $\mathrm{F}_{2}$ as well as their companion atom $\mathrm{Ar}$ which has the ionization potential close to that of $\mathrm{N}_{2}$ and $\mathrm{F}_{2}$. Besides studying the orientationdependent MPI at several peak intensities of the laser pulse, we analyze the HHG spectra, with the emphasis on the effect of multiple molecular orbitals. In Sec. II, we briefly outline the basic equations of TDDFT and discuss the properties of the exchange-correlation potential and the interaction with 
the laser pulse. In Sec. III, we present the details of the computational method including the spatial discretization of the wave functions and the time propagation procedure. We also define the spin orbital ionization probabilities and establish a relation between the spin orbital probabilities and the total ionization probability of the molecule. In Sec. IV, we present the results of our calculations regarding the MPI probabilities and $\mathrm{HHG}$ radiation energies of $\mathrm{N}_{2}, \mathrm{~F}_{2}$, and Ar. Sec. V contains concluding remarks.

\section{THEORY}

The basic equations of TDDFT are the time-dependent one-electron Kohn-Sham equations for spin orbitals $\psi_{n \sigma}(\boldsymbol{r}, t)$ which involve an effective potential $v_{\text {eff, } \sigma}(\boldsymbol{r}, t)$ (atomic units are used):

$$
\begin{gathered}
i \frac{\partial}{\partial t} \psi_{n \sigma}(\boldsymbol{r}, t)=\left[-\frac{1}{2} \boldsymbol{\nabla}^{2}+v_{\mathrm{eff}, \sigma}(\boldsymbol{r}, t)\right] \psi_{n \sigma}(\boldsymbol{r}, t), \\
n=1,2, \ldots, N_{\sigma} .
\end{gathered}
$$

Here $N_{\sigma}\left(=N_{\uparrow}\right.$ or $\left.N_{\downarrow}\right)$ is the total number of electrons for a given spin $\sigma$; the total number of electrons in the system is $N=\Sigma_{\sigma} N_{\sigma}$. The time-dependent effective potential $v_{\text {eff, } \sigma}(\boldsymbol{r}, t)$ is a functional of the electron spin densities $\rho_{\sigma}(\boldsymbol{r}, t)$ which are related to the spin orbitals as follows:

$$
\rho_{\sigma}(\boldsymbol{r}, t)=\sum_{n=1}^{N_{\sigma}}\left|\psi_{n \sigma}(\boldsymbol{r}, t)\right|^{2}
$$

(the summation includes all spin orbitals with the same spin). The potential $v_{\text {eff, } \sigma}(\boldsymbol{r}, t)$ can be written in the general form

$$
v_{\mathrm{eff}, \sigma}(\boldsymbol{r}, t)=v_{\mathrm{n}}(\boldsymbol{r})+v_{\mathrm{H}}(\boldsymbol{r}, t)+v_{\mathrm{xc}, \sigma}(\boldsymbol{r}, t)+v_{\mathrm{ext}}(\boldsymbol{r}, t),
$$

where $v_{\mathrm{n}}(\boldsymbol{r})$ is the electron interaction with the nuclei,

$$
v_{\mathrm{n}}(\boldsymbol{r})=-\frac{Z}{\left|\boldsymbol{R}_{1}-\boldsymbol{r}\right|}-\frac{Z}{\left|\boldsymbol{R}_{2}-\boldsymbol{r}\right|}
$$

with $Z$ being the nuclear charge (we consider homonuclear diatomic molecules only), and $\boldsymbol{R}_{1}$ and $\boldsymbol{R}_{2}$ being the positions of the nuclei (which are assumed to be fixed at their equilibrium positions); $v_{\mathrm{H}}(\boldsymbol{r}, t)$ is the Hartree potential due to electron-electron Coulomb interaction,

$$
\begin{gathered}
v_{\mathrm{H}}(\boldsymbol{r}, t)=\int \frac{\rho\left(\boldsymbol{r}^{\prime}, t\right) d^{3} r^{\prime}}{\left|\boldsymbol{r}-\boldsymbol{r}^{\prime}\right|}, \\
\rho(\boldsymbol{r}, t)=\sum_{\sigma} \rho_{\sigma}(\boldsymbol{r}, t) .
\end{gathered}
$$

In any density-functional calculations, the key role is played by the exchange-correlation (xc) potential $v_{\mathrm{xc}, \sigma}(\boldsymbol{r}, t)$ which must be a functional of the electron density. The exact form of $v_{\mathrm{xc}, \sigma}(\boldsymbol{r}, t)$ is unknown. However, high-quality approximations to the xc potential are becoming available. When these potentials, determined by time-independent ground-state DFT, are used along with TDDFT in the electronic structure calculations, both inner shell and excited states can be calculated rather accurately [22]. In the time-dependent calcu- lations, we adopt the commonly used adiabatic approximation, where the xc potential is calculated with the timedependent density. The adiabatic approximation had many successful applications in the recent studies of atomic and molecular processes in intense external fields [19,22]. In this work, we utilize the $\operatorname{LB} \alpha$ (van Leeuwen-Baerends) xc potential [23],

$$
\begin{aligned}
v_{\mathrm{xc}, \sigma}^{\mathrm{LB} \alpha}(\boldsymbol{r}, t)= & \alpha v_{\mathrm{x}, \sigma}^{\mathrm{LSDA}}(\boldsymbol{r}, t)+v_{\mathrm{c}, \sigma}^{\mathrm{LSDA}}(\boldsymbol{r}, t) \\
& -\frac{\beta x_{\sigma}^{2}(\boldsymbol{r}, t) \rho_{\sigma}^{1 / 3}(\boldsymbol{r}, t)}{1+3 \beta x_{\sigma}(\boldsymbol{r}, t) \ln \left\{x_{\sigma}(\boldsymbol{r}, t)+\left[x_{\sigma}^{2}(\boldsymbol{r}, t)+1\right]^{1 / 2}\right\}} .
\end{aligned}
$$

The $\operatorname{LB} \alpha$ potential contains two parameters, $\alpha$ and $\beta$, which have been adjusted in time-independent DFT calculations of several molecular systems and have the values $\alpha=1.19$ and $\beta=0.01$ [23]. The first two terms in Eq. (7), $v_{\mathrm{x}, \sigma}^{\mathrm{LSDA}}$ and $v_{\mathrm{c}, \sigma}^{\mathrm{LSDA}}$ are the exchange and correlation potentials within the local spin density approximation (LSDA). The last term in Eq. (7) is the gradient correction with $x_{\sigma}(\boldsymbol{r})=\left|\boldsymbol{\nabla} \rho_{\sigma}(\boldsymbol{r})\right| / \rho_{\sigma}^{4 / 3}(\boldsymbol{r})$, which ensures the proper long-range asymptotic behavior $v_{\mathrm{xc}, \sigma}^{\mathrm{LB}} \rightarrow$ $-1 / r$ as $r \rightarrow \infty$. The potential (7) has proved to be reliable in molecular TDDFT studies [20,24]. The correct long-range asymptotic behavior of the $\operatorname{LB} \alpha$ potential is crucial in photoionization problems since it allows to reproduce accurate MO energies, and the proper treatment of the molecular continuum.

The potential $v_{\text {ext }}(\boldsymbol{r}, t)$ in Eq. (3) describes the interaction with the laser field. Using the dipole approximation and the length gauge, it can be expressed as follows:

$$
v_{\mathrm{ext}}(\boldsymbol{r}, t)=(\boldsymbol{F}(t) \cdot \boldsymbol{r}) .
$$

Here $\boldsymbol{F}(t)$ is the electric field strength of the laser field, and the linear polarization is assumed. For the laser pulses with the sine-squared envelope, one has

$$
\boldsymbol{F}(t)=\boldsymbol{F}_{0} \sin ^{2} \frac{\pi t}{T} \sin \omega_{0} t,
$$

where $T$ and $\omega_{0}$ denote the pulse duration and the carrier frequency, respectively; $F_{0}$ is the peak field strength. In our calculations, we used the laser wavelength $800 \mathrm{~nm}\left(\omega_{0}\right.$ $=0.056954$ a.u.) and the sine-squared envelope with 20 optical cycles.

\section{COMPUTATIONAL METHOD}

Before solving the Cauchy problem for the set of equations [Eq. (1)], one has to prepare the initial Kohn-Sham spin orbitals $\psi_{n \sigma}(\boldsymbol{r}, t=0)$. This problem is solved within the framework of the time-independent DFT, using the same $\mathrm{LB} \alpha \mathrm{xc}$ potential and appropriate self-consistent procedure. The wave functions and operators are discretized with the help of the generalized pseudospectral (GPS) method in prolate spheroidal coordinates [25-28]. The prolate spheroidal coordinates $\xi, \eta$, and $\varphi$ are related to the Cartesian coordinates $x, y$, and $z$ as follows [29]:

$$
x=a \sqrt{\left(\xi^{2}-1\right)\left(1-\eta^{2}\right)} \cos \varphi,
$$




$$
\begin{gathered}
y=a \sqrt{\left(\xi^{2}-1\right)\left(1-\eta^{2}\right)} \sin \varphi, \\
z=a \xi \eta \quad(1 \leq \xi<\infty,-1 \leq \eta \leq 1) .
\end{gathered}
$$

In Eq. (10) we assume that the molecular axis is directed along the $z$ axis, and the nuclei are located on this axis at the positions $-a$ and $a$, so the internuclear separation $R=2 a$. The initial spin orbitals $\psi_{n \sigma}(\boldsymbol{r})$ satisfy the set of the timeindependent Kohn-Sham equations where the effective potential $v_{\text {eff, } \sigma}(\boldsymbol{r}) \equiv v_{\text {eff, } \sigma}(\boldsymbol{r}, t=0)$ does not include the interaction with the external field,

$$
\begin{gathered}
{\left[-\frac{1}{2} \nabla^{2}+v_{\mathrm{eff}, \sigma}(\boldsymbol{r})\right] \psi_{n \sigma}(\boldsymbol{r})=\epsilon_{n \sigma} \psi_{n \sigma}(\boldsymbol{r}),} \\
v_{\mathrm{eff}, \sigma}(\boldsymbol{r})=v_{\mathrm{n}}(\boldsymbol{r})+v_{\mathrm{H}}(\boldsymbol{r})+v_{\mathrm{xc}, \sigma}(\boldsymbol{r}), \\
n=1,2, \ldots, N_{\sigma} .
\end{gathered}
$$

In Eq. (11), $\epsilon_{n \sigma}$ are the spin orbital energies. In the prolate spheroidal coordinates, the Coulomb interaction with the nuclei is expressed as follows:

$$
v_{\mathrm{n}}(\xi, \eta)=-\frac{2 Z \xi}{a\left(\xi^{2}-\eta^{2}\right)},
$$

and the kinetic energy operator $\hat{T}$ reads as

$$
\begin{aligned}
\hat{T} \equiv-\frac{1}{2} \nabla^{2}= & -\frac{1}{2 a^{2}} \frac{1}{\left(\xi^{2}-\eta^{2}\right)}\left[\frac{\partial}{\partial \xi}\left(\xi^{2}-1\right) \frac{\partial}{\partial \xi}+\frac{\partial}{\partial \eta}\left(1-\eta^{2}\right) \frac{\partial}{\partial \eta}\right. \\
& \left.+\frac{\xi^{2}-\eta^{2}}{\left(\xi^{2}-1\right)\left(1-\eta^{2}\right)} \frac{\partial^{2}}{\partial \varphi^{2}}\right]
\end{aligned}
$$

For the unperturbed molecule, the projection $m$ of the angular momentum onto the molecular axis is conserved, and the spin orbital $\psi_{n \sigma}(\xi, \eta, \varphi)$ can be represented in a separable form,

$$
\psi_{n \sigma}(\xi, \eta, \varphi)=\psi_{n \sigma m}(\xi, \eta) \exp (\operatorname{im} \varphi)
$$

Then the coordinate $\varphi$ is eliminated, and different kinetic energy operators $\hat{T}_{|m|}$ for different $|m|$ are obtained

$$
\begin{aligned}
\hat{T}_{|m|}= & -\frac{1}{2 a^{2}} \frac{1}{\left(\xi^{2}-\eta^{2}\right)}\left[\frac{\partial}{\partial \xi}\left(\xi^{2}-1\right) \frac{\partial}{\partial \xi}+\frac{\partial}{\partial \eta}\left(1-\eta^{2}\right) \frac{\partial}{\partial \eta}\right. \\
& \left.-\frac{m^{2}}{\xi^{2}-1}-\frac{m^{2}}{1-\eta^{2}}\right] .
\end{aligned}
$$

Note that the exact eigenfunctions $\psi_{j \sigma m}(\xi, \eta)$ have factors $\left(\xi^{2}-1\right)^{|m| / 2}\left(1-\eta^{2}\right)^{|m| / 2}$ which are nonanalytical at nuclei for odd $|m|$. Straightforward numerical differentiation of such functions could result in significant loss of accuracy. Therefore different forms of the kinetic energy operators have been suggested for even and odd $m[25,30]$. Besides treating the unperturbed spin orbitals, this method can be applied also for the case of the laser field linearly polarized along the molecular axis since $m$ is conserved in this case, too. However, for arbitrary orientations of the molecular axis with respect to the polarization of the laser field, the molecular states with different projections of the angular momentum are coupled to each other, and the time-dependent Kohn-Sham spin orbitals $\psi_{n \sigma}(\boldsymbol{r}, t)$ represent a linear combination of quantum states with different $m$. In this case, it is not convenient to have different kinetic energy operators for the parts of the wave function corresponding to even and odd $m$ values. We have solved this problem applying a special coordinate mapping transformation which ensures accurate numerical treatment of possible singularities at the nuclei while using a common discretized kinetic energy operator for the whole wave function.

Since the projection of the electron angular momentum onto the molecular axis is not conserved for arbitrary oriented molecules, there is no reason to separate the parts of the wave function corresponding to definite $m$. Instead, we apply a full $3 \mathrm{D}$ discretization with respect to the coordinates $\xi$, $\eta$, and $\varphi$. For $\xi$ and $\eta$, we use the GPS discretization with nonuniform distribution of the grid points; for $\varphi$, the Fourier grid (FG) method [31] with uniform spacing of the grid points is more appropriate.

The coordinates $\xi$ and $\eta$ are mapped to the interval $[-1,1]$ with the help of the following transformations

$$
\xi=1+R_{l} \frac{(1+x)^{2}}{a\left(1-x+\frac{4 R_{l}}{R_{b}-a}\right)},
$$

$$
\eta=-\cos \left[\frac{\pi}{2}(1+y)\right] \text {. }
$$

Here $R_{l}$ is the mapping parameter; it can be used to adjust the distribution of the grid points thus improving the accuracy of the calculations. Further improvement of the accuracy is achieved by solving the problem in the finite volume which results in a denser grid for the same number of grid points. $R_{b}$ is the boundary value for the $a \xi$ (pseudoradial) grid; we choose it large enough for proper description of the ionization dynamics. Note that with the mapping transformations (17) and (18) applied, the wave functions are analytic functions of $x$ and $y$ at the nuclei for both even and odd projections of the angular momentum. For the GPS discretization of $x$ and $y$ we use the Gauss-Radau and Gauss-Legendre sets of collocation points, respectively. Since the point $x=1$ belongs to the Gauss-Radau set, we can impose the zero boundary condition for the spin orbitals at $\xi=R_{b} / a$ explicitly. The discretized kinetic energy operator takes the form of the matrix $T_{i j k ; i^{\prime} j^{\prime} k^{\prime}}$,

$$
\begin{aligned}
T_{i j k ; i^{\prime} j^{\prime} k^{\prime}}= & \frac{1}{2 a^{2}}\left[\frac{T_{i i^{\prime}}^{(\xi)} \delta_{j j^{\prime}}+T_{j j^{\prime}}^{(\eta)} \delta_{i i^{\prime}}}{\sqrt{\left(\xi_{i}^{2}-\eta_{j}^{2}\right)\left(\xi_{i^{\prime}}^{2}-\eta_{j^{\prime}}^{2}\right)}} \delta_{k k^{\prime}}\right. \\
& \left.+\frac{T_{k k^{\prime}}^{(\varphi)} \delta_{i i^{\prime}} \delta_{j j^{\prime}}}{\left(\xi_{i}^{2}-1\right)\left(1-\eta_{j}^{2}\right)}\right]
\end{aligned}
$$

where the partial matrices $T_{i i^{\prime}}^{(\xi)}, T_{j j^{\prime}}^{(\eta)}$, and $T_{k k^{\prime}}^{(\varphi)}$ related to the coordinates $\xi, \eta$, and $\varphi$, respectively, are defined as follows:

$$
T_{i i^{\prime}}^{(\xi)}=\sqrt{\frac{\left(1+x_{i}\right)\left(1+x_{i^{\prime}}\right)}{\xi_{i}^{\prime} \xi_{i^{\prime}}^{\prime}}} \sum_{n=1}^{N_{x}} \frac{\xi_{n}^{2}-1}{\xi_{n}^{\prime}\left(1+x_{n}\right)} d_{n i}^{x} d_{n i^{\prime}}^{x},
$$




$$
\begin{gathered}
T_{j j^{\prime}}^{(\eta)}=\sqrt{\frac{\left(1-y_{j}^{2}\right)\left(1-y_{j^{\prime}}^{2}\right)}{\eta_{j}^{\prime} \eta_{j^{\prime}}^{\prime}}} \sum_{n=1}^{N_{y}} \frac{1-\eta_{n}^{2}}{\eta_{n}^{\prime}\left(1-y_{n}^{2}\right)} d_{n j}^{y} d_{n j^{\prime}}^{y}, \\
T_{k k^{\prime}}^{(\varphi)}=\frac{2}{N_{\varphi}} \sum_{n=1}^{\left(N_{\varphi^{-1}}\right) / 2} n^{2} \cos \left[\frac{2 \pi n}{N_{\varphi}}\left(k-k^{\prime}\right)\right] .
\end{gathered}
$$

In Eqs. (19)-(22), $x_{i}$ and $y_{j}$ denote the collocation points of the Gauss-Radau and Gauss-Legendre quadrature, respectively,

$$
\begin{gathered}
x_{i}: \quad P_{N_{x}}\left(x_{i}\right)-P_{N_{x}+1}\left(x_{i}\right)=0, \\
y_{j}: \quad P_{N_{y}}\left(y_{j}\right)=0,
\end{gathered}
$$

$P_{l}(z)$ being the Legendre polynomials. $N_{x}$ is the number of grid points for the coordinate $\xi$ (not including the end point $R_{b}$ ), $N_{y}$ is the number of grid points for the coordinate $\eta$, and $N_{\varphi}$ is the number of the (equally spaced) grid points for the coordinate $\varphi ; N_{\varphi}$ must be an odd number for the current implementation of the FG method. The notations $\xi_{i}$ and $\eta_{j}$ stand for the discretized spheroidal coordinates while $\xi_{i}^{\prime}$ and $\eta_{j}^{\prime}$ are used for the derivatives of the mapping functions (17) and (18), respectively, at the grid points. The matrices $d_{i i^{\prime}}^{x}$ and $d_{j j^{\prime}}^{y}$ represent the discretized first derivative operator, using the corresponding (Gauss-Radau and Gauss-Legendre) sets of collocation points,

$$
\begin{gathered}
d_{i i^{\prime}}^{x}=\frac{1}{x_{i}-x_{i^{\prime}}}\left(i \neq i^{\prime}\right), \quad d_{i i}^{x}=-\frac{1}{2\left(1+x_{i}\right)}, \\
d_{N_{x}+1, N_{x}+1}^{x}=\frac{1}{4} N_{x}\left(N_{x}+2\right), \\
d_{j j^{\prime}}^{y}=\frac{1}{y_{j}-y_{j^{\prime}}}\left(j \neq j^{\prime}\right), \quad d_{j j}^{y}=\frac{y_{j}}{1-y_{j}^{2}} .
\end{gathered}
$$

The discretized eigenvalue problem (11) takes the form:

$$
\sum_{i^{\prime} j^{\prime} k^{\prime}}\left[T_{i j k ; i^{\prime} j^{\prime} k^{\prime}}+v_{\mathrm{eff}, \sigma}\left(\xi_{i}, \eta_{j}\right) \delta_{i i^{\prime}} \delta_{j j^{\prime}} \delta_{k k^{\prime}}\right] \phi_{n \sigma ; i^{\prime} j^{\prime} k^{\prime}}=\epsilon_{n \sigma} \phi_{n \sigma ; i j k} \text {. }
$$

Note that the potential terms are diagonal in the GPS and FG methods. They are represented by their values at the discretized coordinates, so no calculation of the potential energy matrix elements is required. The quantities $\phi_{n \sigma ; j k}$ are related to the spin orbital at the discretized coordinates,

$$
\begin{aligned}
\psi_{n \sigma}\left(\xi_{i}, \eta_{j}, \varphi_{k}\right)= & \phi_{n \sigma ; i j k} \\
& \times \frac{\left(N_{x}+1\right) \sqrt{N_{\varphi}}}{\sqrt{4 \pi a^{3}}} \sqrt{\frac{1-y_{j}^{2}}{1+x_{i}}} \frac{P_{N_{x}}\left(x_{i}\right) P_{N_{y}}^{\prime}\left(y_{j}\right)}{\sqrt{\xi_{i}^{\prime} \eta_{j}^{\prime}} \sqrt{\xi_{i}^{2}-\eta_{j}^{2}}},
\end{aligned}
$$

$P_{N_{x}}(x)$ and $P_{N_{y}}^{\prime}(y)$ being the Legendre polynomial and its first derivative, respectively. With the appropriate Gauss quadrature rules used to perform integration with respect to $\xi$ and $\eta$ coordinates, the normalization integral of the spin or- bitals $\psi_{n \sigma}(\xi, \eta, \varphi)$ can be expressed as follows:

$$
\begin{aligned}
\int d^{3} r\left|\psi_{n \sigma}(\boldsymbol{r})\right|^{2}= & a^{3} \int_{1}^{\infty} d \xi \int_{-1}^{1} d \eta \int_{0}^{2 \pi} d \varphi\left(\xi^{2}-\eta^{2}\right) \\
& \times\left|\psi_{n \sigma}(\xi, \eta, \varphi)\right|^{2} \\
= & \sum_{i=1}^{N_{x}} \sum_{j=1}^{N_{y}} \sum_{k=1}^{N_{\varphi}}\left|\phi_{n \sigma ; i j k}\right|^{2} .
\end{aligned}
$$

The time-dependent Kohn-Sham equations [Eq. (1)] are solved by means of the split-operator method with spectral expansion of the propagator matrices previously developed for our $\mathrm{H}_{2}^{+}$calculations $[25,26]$. The initial values for the spin orbitals come from the self-consistent solution of the timeindependent equations [Eq. (11)]. We employ the following split-operator, second-order short-time propagation formula,

$$
\begin{aligned}
\psi_{n \sigma}(\boldsymbol{r}, t+\Delta t)= & \exp \left(-\frac{i}{2} \Delta t \hat{H}_{0}\right) \exp \left[-i \Delta t V\left(\boldsymbol{r}, t+\frac{1}{2} \Delta t\right)\right] \\
& \times \exp \left(-\frac{i}{2} \Delta t \hat{H}_{0}\right) \psi_{n \sigma}(\boldsymbol{r}, t)+O\left((\Delta t)^{3}\right) .
\end{aligned}
$$

Here $\Delta t$ is the time propagation step, $\hat{H}_{0}$ is the unperturbed electronic Hamiltonian which includes the kinetic energy and the effective potential before the laser field switched on,

$$
\hat{H}_{0}=-\frac{1}{2} \nabla^{2}+v_{\mathrm{eff}, \sigma}(\boldsymbol{r}, 0) .
$$

The potential $V(\boldsymbol{r}, t)$ describes the interaction with the laser field and can be expressed as follows:

$$
V(\boldsymbol{r}, t)=v_{\mathrm{eff}, \sigma}(\boldsymbol{r}, t)-v_{\mathrm{eff}, \sigma}(\boldsymbol{r}, 0) .
$$

It contains the direct interaction with the field $v_{\text {ext }}(\boldsymbol{r}, t)$ Eq. (8) as well as terms due to the variation of the density. For the field polarized under the angle $\gamma$ with respect to the molecular axis, the direct interaction can be expressed as follows, using the prolate spheroidal coordinates:

$$
\begin{aligned}
v_{\text {ext }}(\xi, \eta, \varphi, t)= & a F(t)[\xi \eta \cos \gamma \\
& \left.+\sqrt{\left(\xi^{2}-1\right)\left(1-\eta^{2}\right)} \cos \varphi \sin \gamma\right] .
\end{aligned}
$$

The operator $\exp \left(-\frac{i}{2} \Delta t \hat{H}_{0}\right)$ is constructed by the spectral expansion,

$$
\exp \left(-\frac{i}{2} \Delta t \hat{H}_{0}\right)=\sum_{n} \exp \left(-\frac{i}{2} \Delta t E_{n}\right)\left|\psi_{n}\right\rangle\left\langle\psi_{n}\right| .
$$

In practical calculations, the summation in Eq. (34) includes all eigenvectors of Eq. (11) with the energies $E_{n}<E_{b}$ where the upper limit $E_{b}$ should be large enough to describe all relevant physical processes. The high-energy contributions are discarded because for the limited accuracy of the calculations (that is, finite number of grid points), spurious transitions to high-energy states may occur during the time propagation. With the control of high-energy contributions to the propagator matrix, we can avoid population of physically irrelevant regions of the energy spectrum and improve numerical stability of the computations. In the present work, we 
use $E_{b}=5$ a.u.; this is a reasonable value for the carrier frequency and intensities of the laser field used in the calculations.

For the given $\Delta t$, the propagator matrix $\exp \left(-\frac{i}{2} \Delta t \hat{H}_{0}\right)$ is time independent and constructed only once before the propagation process starts. The matrix $\exp [-i \Delta t V(\boldsymbol{r}, t$ $\left.\left.+\frac{1}{2} \Delta t\right)\right]$ is time-dependent and must be calculated at each time step. However, for interaction with the laser field in the length gauge, this matrix is diagonal (as any multiplication by the function of the coordinates in the GPS and FG methods), and its calculation is not time consuming.

The propagation procedure based on Eq. (30) is applied sequentially starting at $t=0$ and ending at $t=T$. As a result, the spin orbitals $\psi_{n \sigma}(\xi, \eta, \varphi, t)$ are obtained on a uniform time grid within the interval $[0, T]$. The space domain is finite with the linear dimension restricted by the end point $R_{b}$. We choose $R_{b}=40$ a.u.; the corresponding space volume contains all relevant physics for the laser field parameters used in the calculations. Between 20 and 40 a.u. we apply an absorber which smoothly brings down the wave function for each spin orbital without spurious reflections. Absorbed parts of the wave packet localized beyond 20 a.u. describe unbound states populated during the ionization process. Because of the absorber, the normalization integrals of the wave functions $\psi_{n \sigma}(\boldsymbol{r}, t)$ decrease in time. Calculated after the pulse, they give the survival probabilities $\mathcal{P}_{n \sigma}^{(\mathrm{s})}$ for each spin orbital,

$$
\mathcal{P}_{n \sigma}^{(\mathrm{s})}=\int d^{3} r\left|\psi_{n \sigma}(\boldsymbol{r}, T)\right|^{2}
$$

Then one can define the spin orbital ionization probabilities $\mathcal{P}_{n \sigma}^{(i)}$ as

$$
\mathcal{P}_{n \sigma}^{(\mathrm{i})}=1-\mathcal{P}_{n \sigma}^{(\mathrm{s})}
$$

We note that the quantities $\mathcal{P}_{n \sigma}^{(\mathrm{s})}$ represent the survival probabilities for the electron occupying the unperturbed $\psi_{n \sigma}(\boldsymbol{r}, t$ $=0)$ spin orbital before the laser pulse. They may not describe the actual population of the same unperturbed spin orbital after the pulse. The final wave function $\psi_{n \sigma}(\boldsymbol{r}, T)$ reflects transitions to other bound states as well, resulting from the interaction with the laser field. Similarly, the electrons originally occupying other unperturbed spin orbitals may exhibit transitions to this particular spin orbital after the pulse. In intense fields or when a resonance condition between any two spin orbitals is satisfied, these effects may be significant. Accordingly, the quantity $\mathcal{P}_{n \sigma}^{(\mathrm{i})}$ represents the ionization probability for the electron originally occupying the unperturbed $\psi_{n \sigma}(\boldsymbol{r}, t=0)$ spin orbital.

The total survival probability $\mathcal{P}^{(\mathrm{s})}$ can be calculated as a product of the spin orbital survival probabilities,

$$
\mathcal{P}^{(\mathrm{s})}=\prod_{n \sigma} \mathcal{P}_{n \sigma}^{(\mathrm{s})}=\prod_{n \sigma}\left(1-\mathcal{P}_{n \sigma}^{(\mathrm{i})}\right) .
$$

Accordingly, the total ionization probability can be written as
TABLE I. Absolute values of spin orbital energies of $\mathrm{N}_{2}, \mathrm{~F}_{2}$, and Ar. (A) Present DFT calculations (eV). (B) Experimental ionization energies $(\mathrm{eV})$.

\begin{tabular}{lccc}
\hline \hline Molecule & Spin-orbital & $\mathrm{A}$ & $\mathrm{B}$ \\
\hline $\mathrm{N}_{2}$ & $2 \sigma_{u}$ & 18.5 & 18.7 (Ref. [33]) \\
& $1 \pi_{u}$ & 16.9 & 17.2 (Ref. [33]) \\
& $3 \sigma_{g}(\mathrm{HOMO})$ & 15.5 & 15.6 (Ref. [33]) \\
$\mathrm{F}_{2}$ & $3 \sigma_{g}$ & 21.9 & 21.0 (Ref. [34]) \\
& $1 \pi_{u}$ & 19.2 & 19.0 (Ref. [34]) \\
& $1 \pi_{g}(\mathrm{HOMO})$ & 16.0 & 15.7 (Ref. [34]) \\
$\mathrm{Ar}$ & $3 s$ & 29.0 & 29.3 (Ref. [35]) \\
& $3 p$ & 15.3 & 15.8 (Ref. [35]) \\
\hline \hline
\end{tabular}

$$
\mathcal{P}^{(\mathrm{i})}=1-\mathcal{P}^{(\mathrm{s})}=1-\prod_{n \sigma}\left(1-\mathcal{P}_{n \sigma}^{(\mathrm{i})}\right) .
$$

The total ionization probability as defined by Eq. (38) reduces to the sum of the spin orbital probabilities only in the limit of the weak laser field ( $\left.\operatorname{small}^{\mathcal{P}_{n \sigma}^{(i)}}\right)$.

\section{RESULTS}

In the calculations, we used the experimental values of the equilibrium internuclear separations for the diatomic molecules [32] (2.074 a.u. for $\mathrm{N}_{2}$ and 2.668 a.u. for $\mathrm{F}_{2}$ ). In Table I, we summarize the energies for the spin orbitals that have a significant contribution to MPI and $\mathrm{HHG}$ and the corresponding experimental vertical ionization energies. Also presented are the data for the companion Ar atom which has an ionization potential close to that of $\mathrm{N}_{2}$ and $\mathrm{F}_{2}$ and is expected to manifest close ionization probabilities as well. The agreement between the calculated and experimental values is fairly good for all three systems indicating a good quality of the $\operatorname{LB} \alpha$ exchange-correlation potential. We note that the original unmodified potential (7) was used in all calculations.

\section{A. Multiphoton ionization}

We have computed the orientation-dependent MPI probabilities for $\mathrm{N}_{2}$ molecule at the peak intensities $1 \times 10^{14}, 2$ $\times 10^{14}$, and $5 \times 10^{14} \mathrm{~W} / \mathrm{cm}^{2}$ (Figs. 1-3). The orientation dependence of the total MPI probability is in a good accord with the experimental observations [6,7] for this molecule and reflects the symmetry of its HOMO: the maximum corresponds to the parallel orientation. However, multielectron effects are quite important for $\mathrm{N}_{2}$, particularly at intermediate orientation angles. In the angle range around $30^{\circ}$, the ionization probability of HOMO-1 $\left(1 \pi_{u}\right)$ is larger than that of HOMO $\left(3 \sigma_{g}\right)$ for all three intensities used in the calculations; the largest difference between the HOMO and HOMO- 1 contributions in the vicinity of $25^{\circ}$ is observed at the intensity $2 \times 10^{14} \mathrm{~W} / \mathrm{cm}^{2}$ (Fig. 2). For the highest intensity $5 \times 10^{14} \mathrm{~W} / \mathrm{cm}^{2}$ (Fig. 3), the ionization probability of HOMO-2 $\left(2 \sigma_{u}\right)$ also exceeds that of HOMO for the angles between $10^{\circ}$ and $40^{\circ}$. Despite the orbital ionization probabilities have local minima and maxima, the total probability 

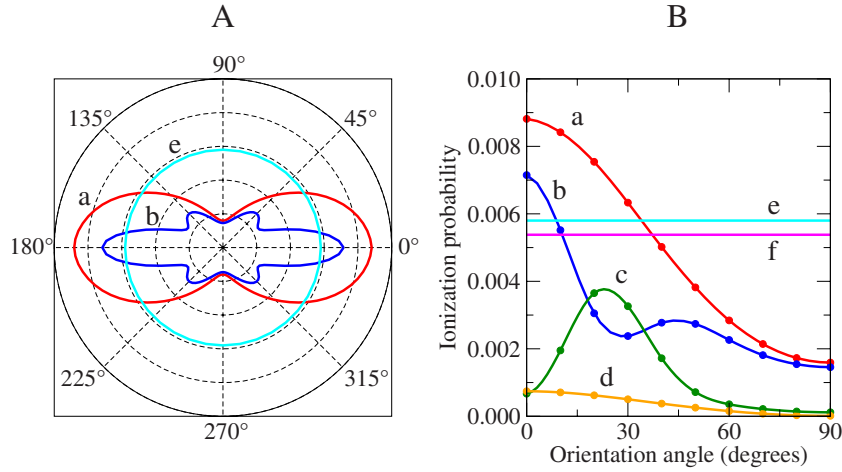

FIG. 1. (Color online) MPI probabilities of $\mathrm{N}_{2}$ molecule and $\mathrm{Ar}$ atom for the peak intensity $1 \times 10^{14} \mathrm{~W} / \mathrm{cm}^{2}$ in polar (panel A) and Cartesian (panel B) coordinates: (a) total probability for $\mathrm{N}_{2}$, (b) $3 \sigma_{g}$ (HOMO) probability for $\mathrm{N}_{2}$, (c) $1 \pi_{u}$ (HOMO-1) probability for $\mathrm{N}_{2}$, (d) $2 \sigma_{u}$ probability for $\mathrm{N}_{2}$, (e) total probability for Ar, (f) $3 p_{0}$ probability for Ar.

shows monotonous dependence on the orientation angle. With increasing the peak intensity of the laser field, the orientation angle distribution of the total ionization probability becomes more isotropic. For comparison, we show also the ionization probability of the Ar atom. As one can see from Figs. 1-3, the absolute values of the ionization probabilities of $\mathrm{N}_{2}$ and $\mathrm{Ar}$ are close to each other. However, the innershell contributions are less important for Ar: the total probability is dominated by the highest-occupied $(3 p)$ shell contribution. An analysis of the spin orbital energies (Table I) can help to understand the relative importance of MPI from the inner shells in $\mathrm{N}_{2}$ compared to that in Ar. The smaller the ionization potential of the electronic shell, the easier it can be ionized. That is why HOMO is generally expected to give the main contribution to the MPI probability. However, in $\mathrm{N}_{2}$ the ionization potential of HOMO-1 is quite close to that of HOMO (the difference between the calculated values is 1.4 $\mathrm{eV}$ ), and in the strong enough laser field both shells show comparable ionization probabilities (a possible resonance between HOMO and HOMO-1 in the $800 \mathrm{~nm}$ laser field also favors that; see discussion of HHG in Sec. IV B below). At
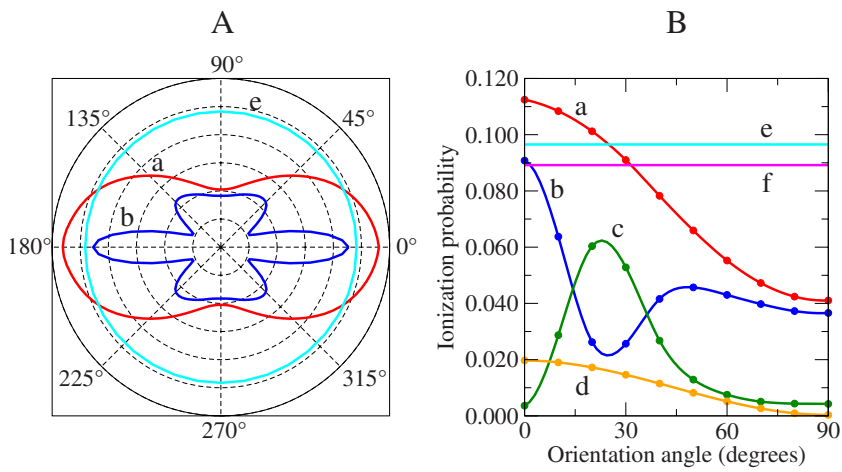

FIG. 2. (Color online) MPI probabilities of $\mathrm{N}_{2}$ molecule and $\mathrm{Ar}$ atom for the peak intensity $2 \times 10^{14} \mathrm{~W} / \mathrm{cm}^{2}$ in polar (panel A) and Cartesian (panel B) coordinates: (a) total probability for $\mathrm{N}_{2}$, (b) $3 \sigma_{g}$ (HOMO) probability for $\mathrm{N}_{2}$, (c) $1 \pi_{u}$ (HOMO-1) probability for $\mathrm{N}_{2}$, (d) $2 \sigma_{u}$ probability for $\mathrm{N}_{2}$, (e) total probability for Ar, (f) $3 p_{0}$ probability for Ar.

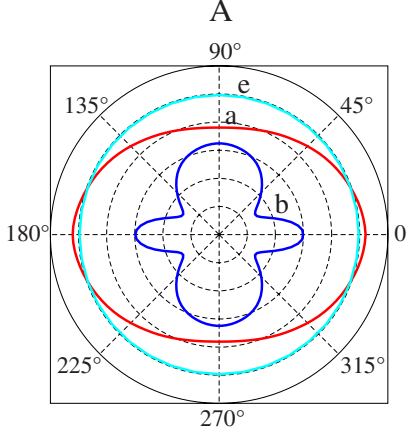

B

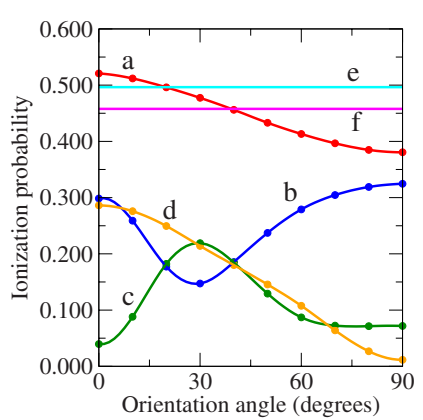

FIG. 3. (Color online) MPI probabilities of $\mathrm{N}_{2}$ molecule and $\mathrm{Ar}$ atom for the peak intensity $5 \times 10^{14} \mathrm{~W} / \mathrm{cm}^{2}$ in polar (panel A) and Cartesian (panel B) coordinates: (a) total probability for $\mathrm{N}_{2}$, (b) $3 \sigma_{g}$ (HOMO) probability for $\mathrm{N}_{2}$, (c) $1 \pi_{u}$ (HOMO-1) probability for $\mathrm{N}_{2}$, (d) $2 \sigma_{u}$ probability for $\mathrm{N}_{2}$, (e) total probability for $\mathrm{Ar}$, (f) $3 p_{0}$ probability for Ar.

the same time, the gap between the $3 p$ and $3 s$ spin orbital energies in $\mathrm{Ar}$ is much larger (our calculation gives the value $13.7 \mathrm{eV}$ ), and the $3 p$ contribution to the MPI probability remains dominant for all three laser intensities.

For $\mathrm{F}_{2}$, the total ionization probability appears smaller than that of $\mathrm{N}_{2}$ (and Ar) at the same laser intensity. We present here the results for the peak intensities $2 \times 10^{14}, 5$ $\times 10^{14}$, and $1 \times 10^{15} \mathrm{~W} / \mathrm{cm}^{2}$ (Figs. 4-6). The ratio of the MPI probabilities of $\mathrm{Ar}$ and $\mathrm{F}_{2}\left(\right.$ at $40^{\circ}$ ) is approximately equal to 4.2 for the intensity $2 \times 10^{14} \mathrm{~W} / \mathrm{cm}^{2}, 1.9$ for 5 $\times 10^{14} \mathrm{~W} / \mathrm{cm}^{2}$, and 1.4 for $1 \times 10^{15} \mathrm{~W} / \mathrm{cm}^{2}$. As one can see, with increasing the intensity of the laser field, the MPI probabilities of all three systems become closer. At the lower intensity $2 \times 10^{14} \mathrm{~W} / \mathrm{cm}^{2}$ (Fig. 4), the pattern for the orientation dependence of MPI in $\mathrm{F}_{2}$ resembles that experimentally observed in $\mathrm{O}_{2}$ [6] since both molecules have the HOMO of the same symmetry $\left(1 \pi_{g}\right)$, and the HOMO contribution is dominant at this intensity. The maximum in the orientation angle distribution of the total MPI probability points at $40^{\circ}$. The HOMO- 1 contribution is less important than that in $\mathrm{N}_{2}$, and this is well explained by the larger gap between the HOMO and HOMO-1 energies $(3.2 \mathrm{eV})$. At the higher intensity $5 \times 10^{14} \mathrm{~W} / \mathrm{cm}^{2}$ (Fig. 5), the overall MPI

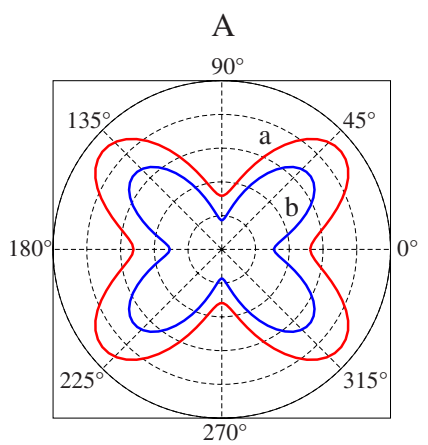

B

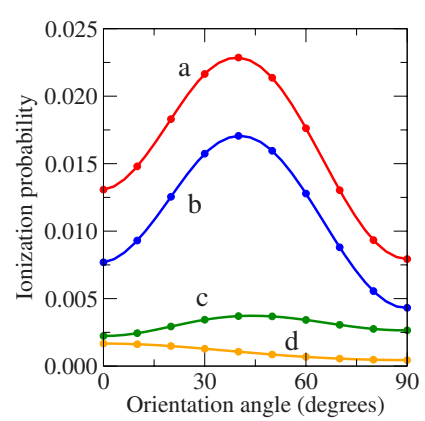

FIG. 4. (Color online) MPI probabilities of $\mathrm{F}_{2}$ molecule for the peak intensity $2 \times 10^{14} \mathrm{~W} / \mathrm{cm}^{2}$ in polar (panel A) and Cartesian (panel B) coordinates: (a) total probability, (b) $1 \pi_{g}$ (HOMO) probability, (c) $1 \pi_{u}$ (HOMO-1) probability, (d) $3 \sigma_{g}$ probability. 

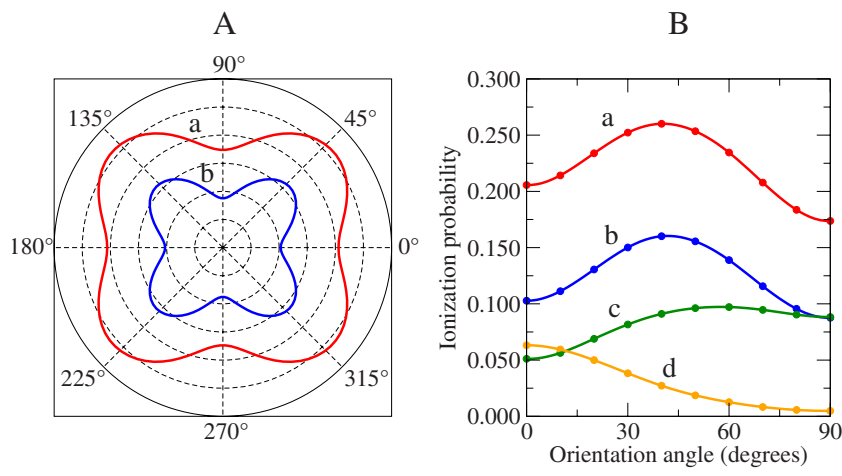

FIG. 5. (Color online) MPI probabilities of $F_{2}$ molecule for the peak intensity $5 \times 10^{14} \mathrm{~W} / \mathrm{cm}^{2}$ in polar (panel A) and Cartesian (panel B) coordinates: (a) total probability, (b) $1 \pi_{g}$ (HOMO) probability, (c) $1 \pi_{u}$ (HOMO-1) probability, (d) $3 \sigma_{g}$ probability.

orientation dependence becomes more isotropic with shallow minima at the angles $\gamma=0^{\circ}$ and $\gamma=90^{\circ}$. The contributions of the inner electronic shells to the total MPI probability become significant. For example, in the vicinity of $\gamma=90^{\circ}$, HOMO and HOMO-1 have equal orbital ionization probabilities; for the parallel orientation $\left(\gamma=0^{\circ}\right)$, HOMO-2 contribution exceeds that of HOMO- 1 , and these two inner shells together contribute as much as HOMO to the total MPI probability.

The picture changes even more dramatically for the highest intensity $1 \times 10^{15} \mathrm{~W} / \mathrm{cm}^{2}$ (Fig. 6). First, the orientation dependence of the total MPI probability becomes almost isotropic. Second, the contribution of HOMO-1 exceeds that of HOMO at all angles, and for small angles $\left(0^{\circ}\right.$ to $\left.18^{\circ}\right)$ both HOMO and HOMO-1 are dominated by HOMO-2 $\left(3 \sigma_{g}\right)$. Thus with increasing the laser intensity, we observe the same orbital switching as for $\mathrm{N}_{2}$ at the intensity $5 \times 10^{14} \mathrm{~W} / \mathrm{cm}^{2}$ where the HOMO-2 becomes dominant at intermediate orientation angles. This significant and interesting phenomenon was reported previously for the parallel orientation of the molecular axis [20]. We are aware of the recent research [36] that reported the absence of the orbital switching. However, the work [36] is based on the static model potential ap-
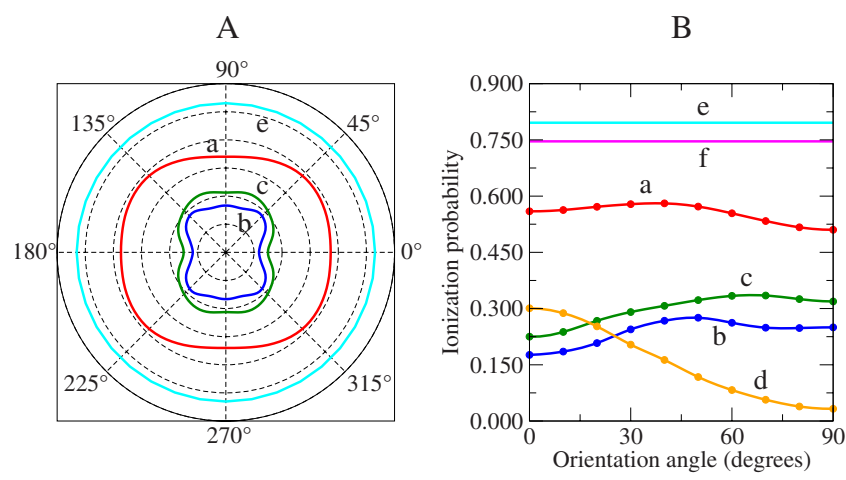

FIG. 6. (Color online) MPI probabilities of $\mathrm{F}_{2}$ molecule and $\mathrm{Ar}$ atom for the peak intensity $1 \times 10^{15} \mathrm{~W} / \mathrm{cm}^{2}$ in polar (panel A) and Cartesian (panel B) coordinates: (a) total probability for $\mathrm{F}_{2}$, (b) $1 \pi_{g}$ (HOMO) probability for $\mathrm{F}_{2}$, (c) $1 \pi_{u}$ (HOMO-1) probability for $\mathrm{F}_{2}$, (d) $3 \sigma_{g}$ probability for $\mathrm{F}_{2}$, (e) total probability for $\mathrm{Ar}$, (f) $3 p_{0}$ probability for Ar. proach, that is, unlike TDDFT, no dynamic response of the electron density to the laser pulse was taken into account. Besides that, it adopted the molecular strong-field approximation which does not take into account the correlated multielectron effects. Thus the conclusions made in Ref. [36] may be subject to the limitations of the method used in that study.

\section{B. High-order harmonic generation}

To calculate the HHG spectra, we employ the widely used semiclassical approach, where the basic expressions come from the classical electrodynamics but the classical quantities such as dipole moment and its acceleration are replaced with the corresponding quantum expectation values. For nonmonochromatic fields (as in our case), the spectral density of the radiation energy emitted for all the time is given by the following expression [37]:

$$
S(\omega)=\frac{4 \omega^{4}}{6 \pi c^{3}}|\widetilde{\boldsymbol{D}}(\omega)|^{2} .
$$

Here $\omega$ is the frequency of radiation, $c$ is the velocity of light, and $\widetilde{\boldsymbol{D}}(\omega)$ is a Fourier transform of the time-dependent dipole moment,

$$
\widetilde{\boldsymbol{D}}(\omega)=\int_{-\infty}^{\infty} d t \boldsymbol{D}(t) \exp (i \omega t)
$$

The time-dependent dipole moment is evaluated as an expectation value of the electron radius-vector with the timedependent total electron density $\rho(\boldsymbol{r}, t)$,

$$
\boldsymbol{D}(t)=\int d^{3} r \boldsymbol{r} \rho(\boldsymbol{r}, t) .
$$

The total energy $\mathcal{E}$ emitted in the harmonic radiation can be calculated by integration of $S(\omega)$,

$$
\mathcal{E}=\int_{0}^{\infty} d \omega S(\omega)
$$

For a long enough laser pulse, the radiation energy spectrum (39) contains peaks corresponding to odd harmonics of the carrier frequency $\omega_{0}$. We define the energy $\mathcal{E}\left(N_{\mathrm{h}}\right)$ emitted in the $N_{\mathrm{h}}$ th harmonic ( $N_{\mathrm{h}}$ is an odd integer number) as follows:

$$
\mathcal{E}\left(N_{\mathrm{h}}\right)=\int_{\left(N_{\mathrm{h}}-1\right) \omega_{0}}^{\left(N_{\mathrm{h}}+1\right) \omega_{0}} d \omega S(\omega) .
$$

Besides the total induced dipole moment $\boldsymbol{D}(t)$, one can also calculate the spin orbital contributions to this quantity replacing the total density $\rho(\boldsymbol{r}, t)$ in Eq. (41) with the spin orbital density $\left|\psi_{n \sigma}(\boldsymbol{r}, t)\right|^{2}$. Unlike the total dipole moment, the total harmonic energy (43) is not equal to the sum of the spin orbital contributions due to the interference terms in Eq. (39).

In Figs. 7 and 8 we present the HHG data for $\mathrm{N}_{2}$ and $\mathrm{F}_{2}$ molecules, respectively, at the peak intensity 2 $\times 10^{14} \mathrm{~W} / \mathrm{cm}^{2}$. To show the orientation dependence of the HHG spectra, we choose three values of the orientation angle 


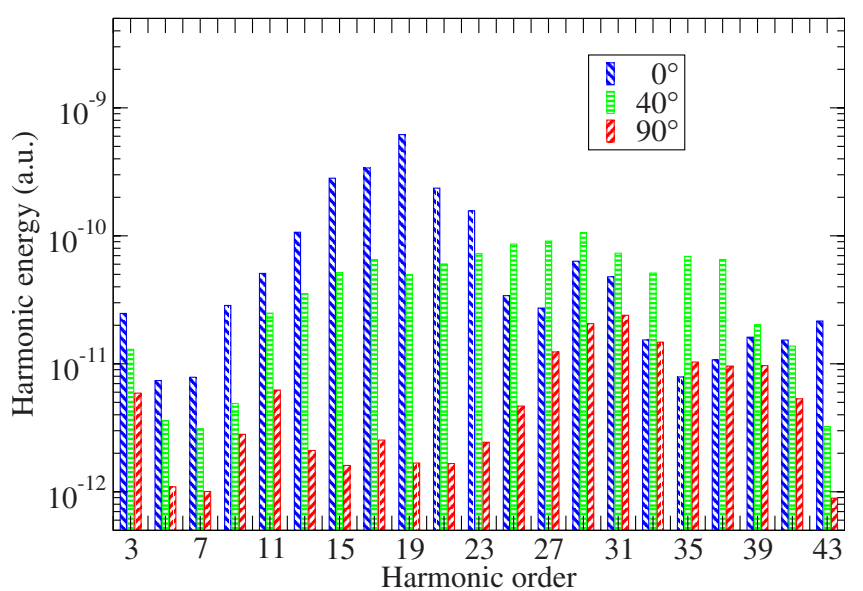

FIG. 7. (Color online) Energy emitted in harmonic radiation by $\mathrm{N}_{2}$ molecule for the peak intensity $2 \times 10^{14} \mathrm{~W} / \mathrm{cm}^{2}$ : left (blue) bar, orientation angle $\gamma=0^{\circ}$; middle (green) bar, orientation angle $\gamma$ $=40^{\circ}$; right $\left(\right.$ red) bar, orientation angle $\gamma=90^{\circ}$.

$\gamma: 0^{\circ}, 40^{\circ}$, and $90^{\circ}$ which represent the limiting cases of the parallel and perpendicular orientation as well as the intermediate angle case. According to the semiclassical recollision model of HHG [38], the cutoff beginning position in the harmonic spectrum is expected at the energy $E_{\mathrm{i}}+3.17 U_{p}$, with $E_{\mathrm{i}}$ and $U_{p}$ being the ionization energy and the ponderomotive potential, respectively. For the intensity 2 $\times 10^{14} \mathrm{~W} / \mathrm{cm}^{2}$ and the wavelength $800 \mathrm{~nm}$, the predicted cutoff position corresponds to the harmonic order 35 . This value is in fairly good agreement with the computed data (Figs. 7 and 8) for the orientation angles $\gamma=40^{\circ}$ and $\gamma$ $=90^{\circ}$; for the parallel orientation $\left(\gamma=0^{\circ}\right)$, the computed HHG spectra extend to slightly higher energies and do not show a clear cutoff at the predicted position.

For all three orientations, the HHG signal from $\mathrm{N}_{2}$ is about an order of magnitude stronger than that from $\mathrm{F}_{2}$; this observation is consistent with the MPI results of Sec. IV A: at this intensity, the MPI signal from $\mathrm{F}_{2}$ is four to ten times weaker than that from $\mathrm{N}_{2}$, depending on the orientation. The

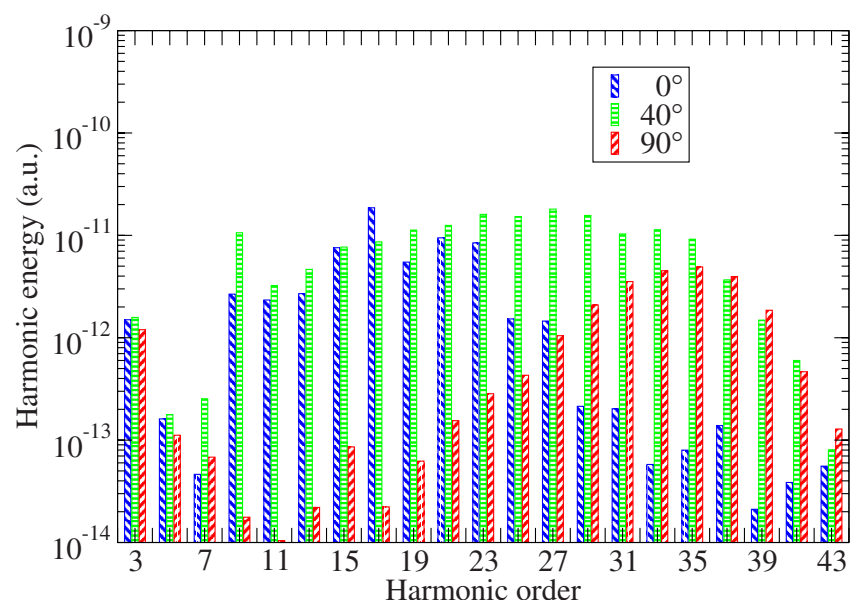

FIG. 8. (Color online) Energy emitted in harmonic radiation by $\mathrm{F}_{2}$ molecule for the peak intensity $2 \times 10^{14} \mathrm{~W} / \mathrm{cm}^{2}$ : left (blue) bar, orientation angle $\gamma=0^{\circ}$; middle (green) bar, orientation angle $\gamma$ $=40^{\circ}$; right (red) bar, orientation angle $\gamma=90^{\circ}$.
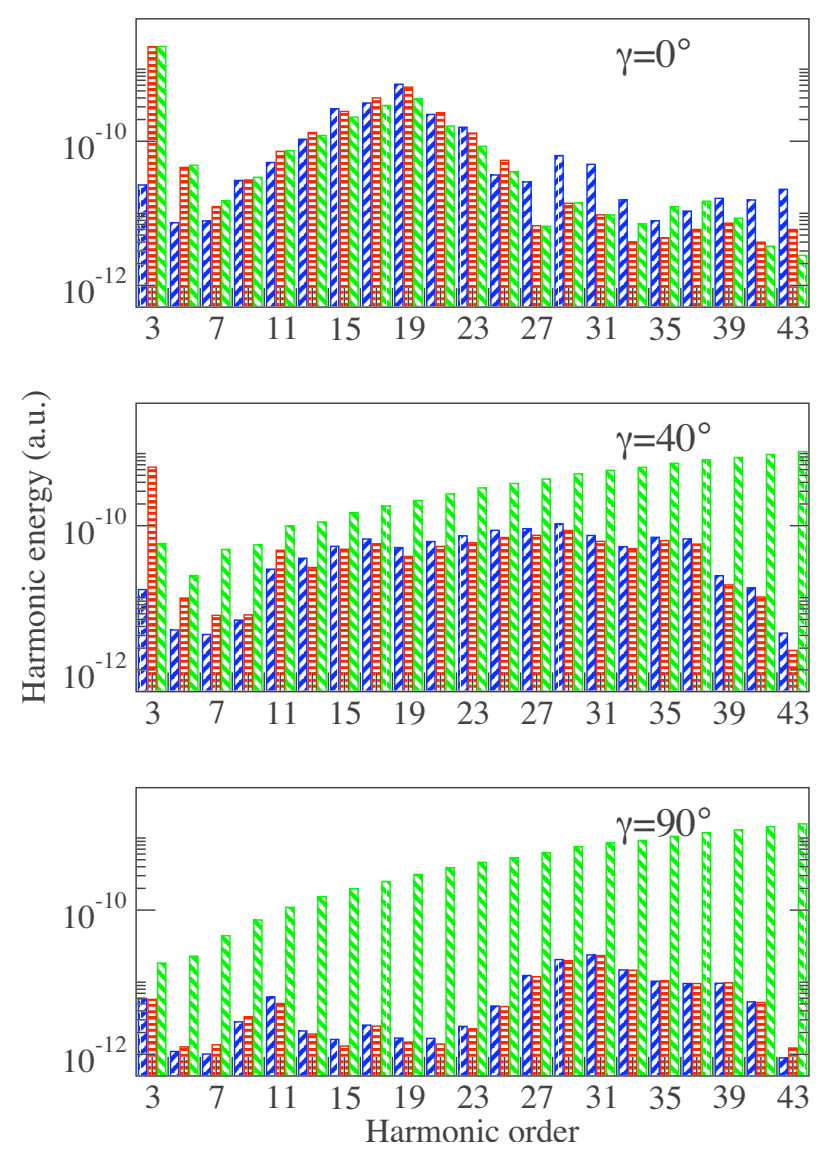

FIG. 9. (Color online) Energy emitted in harmonic radiation by $\mathrm{N}_{2}$ molecule for the peak intensity $2 \times 10^{14} \mathrm{~W} / \mathrm{cm}^{2}$ : upper panel, orientation angle $\gamma=0^{\circ}$; middle panel, orientation angle $\gamma=40^{\circ}$; lower panel, orientation angle $\gamma=90^{\circ}$. The left (blue) bar for each harmonic shows the actual radiation energy while the right (green) bar shows the spectrum that would be a response of HOMO only. The middle (red) bar corresponds to the harmonic spectrum that would be produced by HOMO and HOMO-1 together.

orientation dependence of HHG also resembles that of MPI: HHG is more intense for the orientations where MPI reaches its maximum. It is clearly seen for $F_{2}$ where the radiation energy at $40^{\circ}$ exceeds that at other orientations for almost every harmonic. For $\mathrm{N}_{2}$, the HHG signal at $0^{\circ}$ is dominant in the low-order part of the spectrum whereas in the central part a stronger signal is observed at $40^{\circ}$. One can also see that the emission of the harmonic radiation at the perpendicular orientation $\left(\gamma=90^{\circ}\right)$ is suppressed for both $\mathrm{N}_{2}$ and $\mathrm{F}_{2}$ in the low-order and central parts of the HHG spectra. The maximum in the harmonic energy distribution at $90^{\circ}$ is shifted to higher orders. This result is in a good accord with the recent experimental measurements on $\mathrm{N}_{2}$ [9]. In Ref. [9] it was explained by the dominant contribution from HOMO-1 to the $\mathrm{HHG}$ signal at $\gamma=90^{\circ}$. Our calculations also reveal significant presence of HOMO-1 and other inner $\mathrm{MO}$ in the HHG spectra, and not only at the perpendicular orientation.

To analyze the importance of multielectron effects in HHG, we compare in Figs. 9 and 10 the actual energy emitted in the harmonic radiation with the energy that would be produced by HOMO alone. As one can see, the first few harmonics (3 to 7) always manifest strong involvement of 

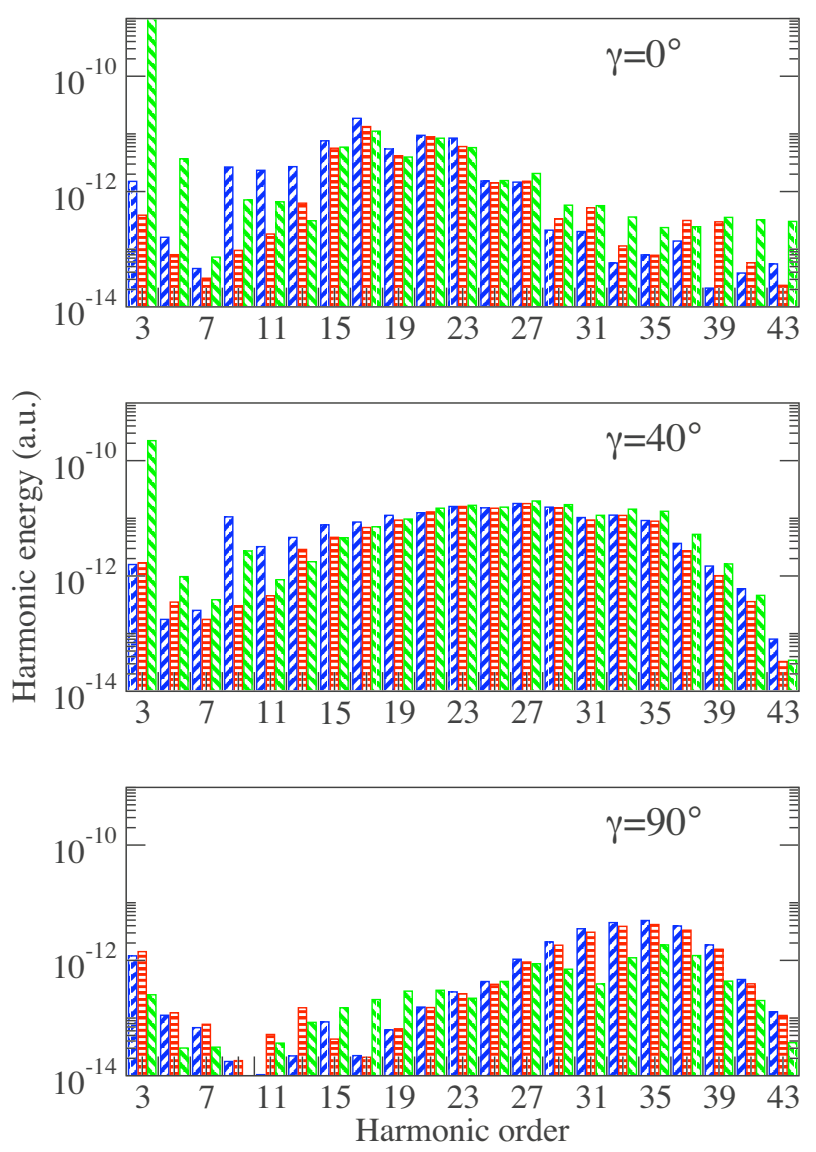

FIG. 10. (Color online) Energy emitted in harmonic radiation by $\mathrm{F}_{2}$ molecule for the peak intensity $2 \times 10^{14} \mathrm{~W} / \mathrm{cm}^{2}$ : upper panel, orientation angle $\gamma=0^{\circ}$; middle panel, orientation angle $\gamma=40^{\circ}$; lower panel, orientation angle $\gamma=90^{\circ}$. The left (blue) bar for each harmonic shows the actual radiation energy while the right (green) bar shows the spectrum that would be a response of HOMO only. The middle (red) bar corresponds to the harmonic spectrum that would be produced by HOMO and HOMO-1 together.

the inner electronic shells. The interference is mostly destructive, so the total radiation energy appears less than that resulting from $\mathrm{HOMO}$ only. However, for $\mathrm{F}_{2}$ at $\gamma=90^{\circ}$ (Fig. 10) the contributions from different spin orbitals interfere constructively, and the result exceeds the HOMO-only radiation energy. For $\mathrm{N}_{2}$ (Fig. 9), the central part of the HHG spectrum at the parallel orientation $\left(\gamma=0^{\circ}\right)$ suggests that the HOMO contribution is dominant; multielectron effects become more important for higher harmonic orders (27 to 43). However, at the orientation angles $\gamma=40^{\circ}$ and $\gamma=90^{\circ}$, the HOMO-only radiation energy is considerably larger than the actual HHG output. These results imply a strong destructive interference between HOMO and the inner shells and indicate that HOMO alone may not provide a correct description of the HHG process. The reason for such a behavior of the HHG spectra lies in the resonance nature of the process. The photon energy corresponding to the carrier frequency of the laser field is $1.55 \mathrm{eV}$, and the energy difference between HOMO $\left(3 \sigma_{g}\right)$ and HOMO-1 $\left(1 \pi_{u}\right)$ in $\mathrm{N}_{2}$ is about $1.4 \mathrm{eV}$. Thus the process takes place in the vicinity of the one-photon resonance between HOMO and HOMO-1. However, because of the selection rules, the $3 \sigma_{g}$ and $1 \pi_{u}$ states can be coupled

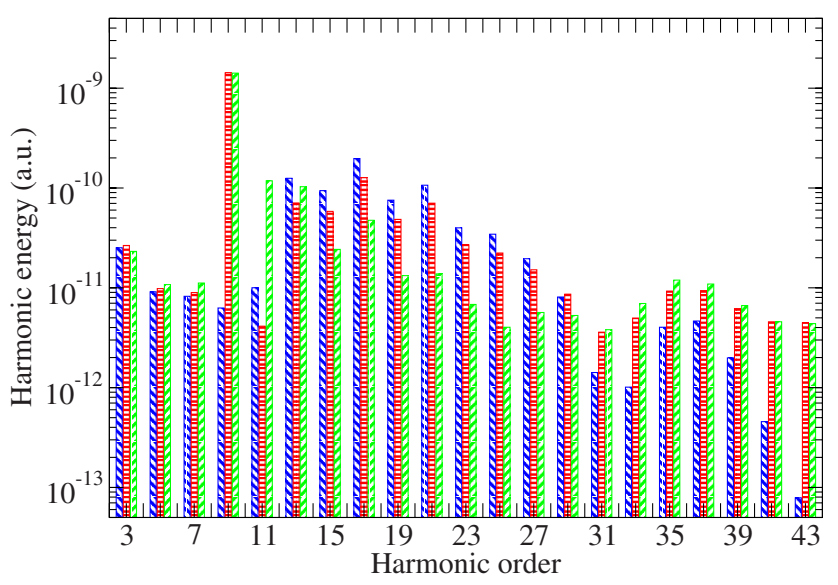

FIG. 11. (Color online) Energy emitted in harmonic radiation by Ar atom for the peak intensity $2 \times 10^{14} \mathrm{~W} / \mathrm{cm}^{2}$. The left (blue) bar for each harmonic shows the actual radiation energy while the right (green) bar shows the spectrum that would be a response of the $3 p_{0}$ orbital only. The middle (red) bar corresponds to the harmonic spectrum that would be produced by the entire $3 p$ shell.

by the linearly polarized laser field at nonparallel orientations only. Thus the resonance is present at $\gamma=40^{\circ}$ and $\gamma$ $=90^{\circ}$ and absent at $\gamma=0^{\circ}$. In the vicinity of the resonance, HOMO and HOMO-1 are strongly coupled to each other, and one must consider their combined response to the laser pulse. As Fig. 9 shows, this combined response results in a radiation energy distribution which appears a good approximation for the total multielectron HHG spectrum.

The gap between the HOMO and HOMO- 1 energies in $\mathrm{F}_{2}$ is about $3.2 \mathrm{eV}$, and the resonance condition is not satisfied. The HOMO HHG spectrum appears a reasonable approximation for the actual radiation energy distribution at all three orientations presented in Fig. 10. Addition of the HOMO-1 response generally improves the agreement with the total HHG spectrum, particularly at $\gamma=90^{\circ}$. A large deviation from the total spectrum for the harmonic orders 9 to 13 at other orientations may be attributed to the resonantly enhanced contributions from the inner $3 \sigma_{g}$ and $2 \sigma_{u}$ spin orbitals.

For comparison with the diatomic molecules, we show in Fig. 11 the HHG spectrum from the Ar atom. The MPI probability of $\mathrm{Ar}$ (see the previous subsection) shows a dominant contribution from the outer $3 p_{0}$ electron which has a zero angular momentum projection onto the laser polarization direction, so the Ar atom may be considered as a single-activeelectron atom in the MPI process. Surprisingly, the multielectron effects appear quite important in the HHG process. The central and high-energy parts of the spectrum show significant contributions from the other electrons in the $3 p$ shell as well as from the inner $3 s$ shell. The interference of different contributions is mostly constructive in the central part of the spectrum and destructive in the high-energy part. A large deviation of the total radiation energy from that produced by the $3 p$ shell only at the ninth harmonic appears due to the nine-photon resonance between the $3 p$ and $3 s$ shells; a correct description of the HHG in this region of the spectrum requires taking into account the $3 s$ contribution. The total HHG spectrum has a clear minimum at the $33 \mathrm{rd}$ harmonic 


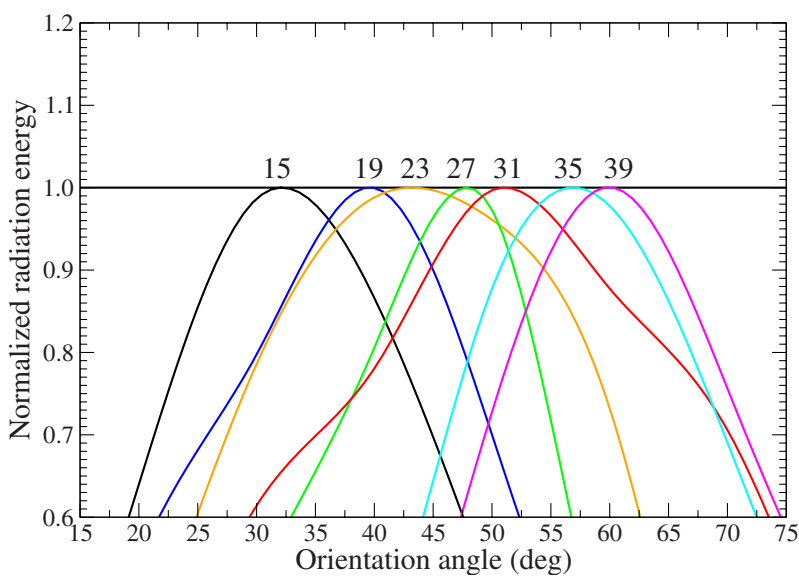

FIG. 12. (Color online) Normalized energy emitted in harmonic radiation by $\mathrm{F}_{2}$ molecule for the peak intensity $2 \times 10^{14} \mathrm{~W} / \mathrm{cm}^{2} \mathrm{vs}$ the orientation angle. For each harmonic, the radiation energy is normalized to unity at the maximum; the curves are marked by the harmonic order.

$(51 \mathrm{eV})$ while the HHG from the $3 p$ shell only exhibits the same minimum at the 31 st harmonic $(48 \mathrm{eV})$. This is a manifestation of the well-known Cooper minimum in Ar. In the photoionization cross section of Ar it was measured at $48 \mathrm{eV}$

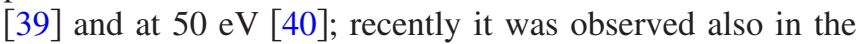
experimental HHG spectra approximately at the same energy [41]. As one can see from Fig. 11, accounting for the innershell contributions to the HHG spectrum slightly shifts the position of the minimum toward higher energies. Note that the one-electron model calculations [42] give the position of the Cooper minimum at the lower energy of $40 \mathrm{eV}$. In the vicinity of the Cooper minimum, the contribution from the $3 p$ shell to the Fourier-transformed dipole moment [Eq. (40)] changes its sign relative to that of the $3 s$ contribution, and this sign change is responsible for switching of the interference pattern from constructive in the central part of the spectrum to destructive for the harmonic orders 31 and higher.

The HHG spectra of diatomic molecules manifest interference effects related to the two-center nature of the molecules. A simplified one-electron two-center recollision model of the molecular HHG results in a straightforward condition for the interference minima or maxima [43],

$$
\cos \gamma=\frac{\pi n}{R \sqrt{2 E_{\mathrm{k}}}}, \quad n=1,2,3 \ldots
$$

where $E_{\mathrm{k}}$ is the kinetic energy of the recolliding electron. Provided all the kinetic energy of the electron is converted into the harmonic radiation energy upon recollision, the condition for the first $(n=1)$ extremum in the series (44) can be recast in the following form:

$$
\cos \gamma=\frac{\pi}{R \sqrt{2 N_{\mathrm{h}} \omega_{0}}} .
$$

Here $N_{\mathrm{h}}$ is the harmonic order (an odd integer number), and the dispersion relationship $E_{\mathrm{k}}=N_{\mathrm{h}} \omega_{0}$ is taken into account. If the electronic $\mathrm{MO}$ can be approximated as a symmetric combination of the atomic orbitals,

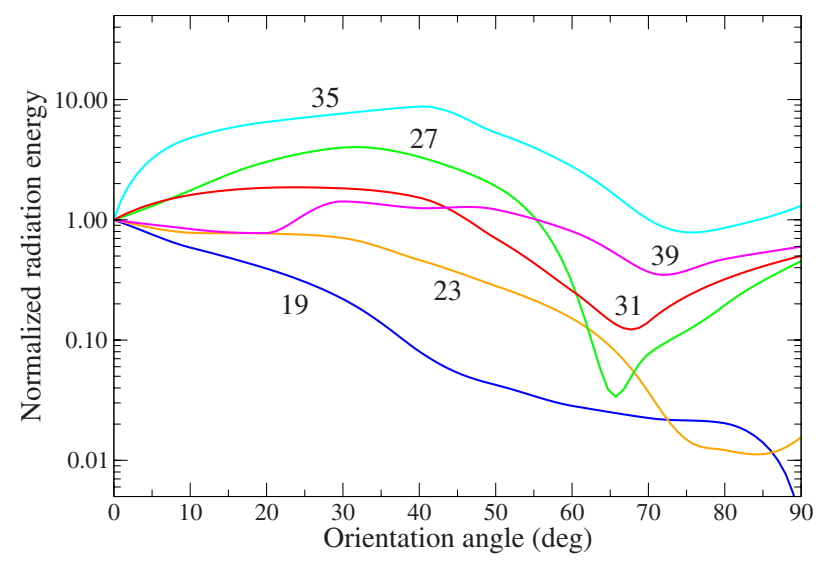

FIG. 13. (Color online) Normalized energy emitted in harmonic radiation by $\mathrm{N}_{2}$ molecule for the peak intensity $2 \times 10^{14} \mathrm{~W} / \mathrm{cm}^{2} \mathrm{vs}$ the orientation angle. For each harmonic, the radiation energy is normalized to unity at $\gamma=0^{\circ}$; the curves are marked by the harmonic order.

$$
\Psi_{\mathrm{m}}^{+}(\boldsymbol{r})=\psi_{\mathrm{a}}\left(\boldsymbol{r}-\frac{1}{2} \boldsymbol{R}\right)+\psi_{\mathrm{a}}\left(\boldsymbol{r}+\frac{1}{2} \boldsymbol{R}\right),
$$

then the extremum (45) is a minimum. In the case of an antisymmetric combination of the atomic orbitals,

$$
\Psi_{\mathrm{m}}^{-}(\boldsymbol{r})=\psi_{\mathrm{a}}\left(\boldsymbol{r}-\frac{1}{2} \boldsymbol{R}\right)-\psi_{\mathrm{a}}\left(\boldsymbol{r}+\frac{1}{2} \boldsymbol{R}\right),
$$

the extremum (45) represents a maximum. Note that not all harmonics in the HHG spectra exhibit the orientationdependent two-center interference minima or maxima: the right-hand side of Eq. (44) must be less than unity at least. For $\mathrm{N}_{2}$ and $\mathrm{F}_{2}$ molecules subject to the $800 \mathrm{~nm}$ laser field with the intensity $2 \times 10^{14} \mathrm{~W} / \mathrm{cm}^{2}$, at their equilibrium internuclear separation, only the first extremum (45) can be important for the two-center interference analysis. In our previous $\mathrm{HHG}$ calculations of the one-electron molecule $\mathrm{H}_{2}^{+}$ [25], we tested Eq. (45) against accurate numerical results and got a fairly good agreement.

For multielectron diatomic molecules, one can try using Eq. (45) based on their HOMO symmetry. For $F_{2}$, HOMO is $1 \pi_{g}$ and satisfies the symmetry of Eq. (47). Thus one can expect maxima in the orientation dependence of the radiation energy. Indeed, our calculations reveal such maxima for the harmonics in the central part of the HHG spectrum. In Fig. 12 , we show the radiation energy for several harmonics in the range 15 to 39 . For better presentation, the radiation energy has been normalized to unity at the maximum for each harmonic. One can see that the orientation angle corresponding to the maximum is shifted toward larger values as the harmonic order is increased, in accordance with Eq. (45). The numerical values of the orientation angle corresponding to the interference maximum are summarized in Table II. As one can see, the agreement between the present TDDFT results and the two-center model (45) is good for the harmonics in the middle of the spectrum (23 to 31 ) and becomes less accurate for the low-order and high-order harmonics. These results agree well with the recent measurements [44] which 
TABLE II. Orientation angles of interference maxima for several harmonics in the $\mathrm{F}_{2}$ HHG spectrum for the peak laser intensity $2 \times 10^{14} \mathrm{~W} / \mathrm{cm}^{2}$.

\begin{tabular}{lccccccc}
\hline \hline & \multicolumn{7}{c}{ Harmonic order } \\
\cline { 2 - 7 } & 15 & 19 & 23 & 27 & 31 & 35 & 39 \\
\hline Present work & $32^{\circ}$ & $40^{\circ}$ & $43^{\circ}$ & $48^{\circ}$ & $51^{\circ}$ & $57^{\circ}$ & $60^{\circ}$ \\
Eq. (45) & $26^{\circ}$ & $37^{\circ}$ & $43^{\circ}$ & $48^{\circ}$ & $51^{\circ}$ & $54^{\circ}$ & $56^{\circ}$ \\
\hline \hline
\end{tabular}

have shown that the dispersion relationship between the kinetic energy of the recolliding electron and the harmonic frequency used in Eq. (45) $\left(E_{\mathrm{k}}=N_{\mathrm{h}} \omega_{0}\right)$ is better satisfied in the central part of the HHG spectrum while for both loworder and high-order harmonics the deviations have been observed. In general, Eq. (45) provides a reasonable description of the two-center interference effects in $\mathrm{F}_{2}$.

The situation is different for the $\mathrm{N}_{2}$ molecule. The symmetry of HOMO $\left(3 \sigma_{g}\right)$ corresponds to Eq. (46) and suggests that the orientation-dependent harmonic radiation exhibits an interference minimum according to Eq. (45). The results of our calculations are shown in Fig. 13 for several harmonics in the range 19 to 39. Since the internuclear separation $R$ for $\mathrm{N}_{2}$ is shorter than that for $\mathrm{F}_{2}$, the minimum can be observed for the harmonic order higher than 21. As it follows from Eq. (45), for the harmonic orders 23 to 39, the orientation angle corresponding to the minimum varies from $21^{\circ}$ to $44^{\circ}$. As one can see from Fig. 13, this is not the case. Harmonics 27 to 39 do have a minimum but it is located at much larger angles, in the interval $65^{\circ}$ to $75^{\circ}$. Thus the formula (45) being successful in the case of the $\mathrm{F}_{2}$ molecule does not provide a correct description of the interference phenomena in $\mathrm{N}_{2}$. We attribute this striking difference in behavior of the two molecules to the resonance nature of the $\mathrm{HHG}$ process in $\mathrm{N}_{2}$. The resonantly enhanced induced time-dependent dipole moment of the molecule significantly affects HHG, that ultimately means a breakdown of the simple one-electron twocenter recollision model. Previously, a failure of Eq. (45) to correctly describe the $\mathrm{HHG}$ interference in $\mathrm{N}_{2}$ was detected in Ref. [45] and explained as a result of substantial contributions to HOMO from the atomic orbitals with different symmetries.

\section{CONCLUSION}

In this paper, we have presented $a b$ initio all-electron TDDFT calculations of MPI and HHG in diatomic molecules $\mathrm{N}_{2}$ and $\mathrm{F}_{2}$ with arbitrary orientation of the molecular axis with respect to the polarization of the laser field. Both processes are affected by the electronic structure of the molecules and symmetries of their HOMO and inner-shell molecular orbitals. We have shown that for the $\mathrm{N}_{2}$ molecule subject to 800 $\mathrm{nm}$ laser pulses with the moderate peak intensity 1 $\times 10^{14} \mathrm{~W} / \mathrm{cm}^{2}$, the contributions of the inner shells the MPI probability are quite significant, particularly at intermediate orientation angles. Multielectron effects in MPI become more pronounced at the higher intensity $2 \times 10^{14} \mathrm{~W} / \mathrm{cm}^{2}$. For the same intensity, the HOMO contribution is still domi- nant for the $\mathrm{F}_{2}$ molecule at all orientations. These observations are in agreement with the electronic structure and positions of the spin orbital energy levels for these species: the gap between the HOMO and HOMO-1 energies in $\mathrm{F}_{2}$ is larger than that in $\mathrm{N}_{2}$. With further increasing the intensity, the effect of the inner-shell electrons on MPI generally becomes more important, and orbital switching may occur: the contributions of spin orbitals with larger ionization potentials exceed those of spin orbitals with smaller ionization potentials. We can see such a behavior in $\mathrm{F}_{2}$ at the highest intensity $1 \times 10^{15} \mathrm{~W} / \mathrm{cm}^{2}$ used in the calculations. Note that for the companion Ar atom the contribution of the highestoccupied $3 p_{0}$ orbital (with the zero angular momentum projection onto the field polarization direction) remains dominant for all intensities; this is well explained by the large difference between the $3 p$ and $3 s$ orbital energies. Another observation is related to the orientation angle dependence of the total MPI probability of $\mathrm{N}_{2}$ and $\mathrm{F}_{2}$ : with increasing the intensity of the laser field it becomes less anisotropic. Several spin orbitals possessing different symmetries make substantial contributions to MPI, and that results in a more uniform distribution of the total MPI probability with respect to the orientation of the molecular axis.

The HHG spectra are influenced by the multielectron effects even more than the MPI probabilities. The high-order harmonics are very sensitive to the interference of the contributions from multiple electronic shells. Even tightly bound electrons which do not contribute much in the MPI process make their presence in the HHG process. In part this is related to possible resonances between different electronic shells. Such a one-photon resonance exists between HOMO and HOMO-1 in $\mathrm{N}_{2}$ for the laser field parameters used in the calculations. Under these conditions, the response of HOMO alone to the laser pulse does not provide a correct description of the HHG spectrum. HOMO and HOMO-1 are strongly coupled by the laser field at nonparallel orientations, and only a coherent sum of their contributions gives a reasonable approximation for the harmonic radiation energy. The interference of the contributions is destructive in this case; in other cases it can be constructive, as one could see in the HHG spectra of $\mathrm{F}_{2}, \mathrm{Ar}$ or $\mathrm{N}_{2}$ at the parallel orientation. In any case, the account of the multielectron effects can change the resulting harmonic radiation energy by orders of magnitude, as compared with the HHG spectrum produced by HOMO only. Some well-understood phenomena such as two-center interference in the HHG spectra of homonuclear diatomic molecules are also affected by the contributions from multiple molecular orbitals with different symmetries; in particular, they may be responsible for the failure of the simple one-electron recollision model in the case of the $\mathrm{N}_{2}$ molecule.

\section{ACKNOWLEDGMENTS}

This work was partially supported by the Chemical Sciences, Geosciences and Biosciences Division of the Office of Basic Energy Sciences, Office of Sciences, U. S. Department of Energy and by the U. S. National Science Foundation. 
[1] F. Krausz and M. Ivanov, Rev. Mod. Phys. 81, 163 (2009).

[2] P. B. Corkum and F. Krausz, Nat. Phys. 3, 381 (2007).

[3] J. H. Posthumus, Rep. Prog. Phys. 67, 623 (2004).

[4] E. Wells, M. J. DeWitt, and R. R. Jones, Phys. Rev. A 66, 013409 (2002).

[5] J. Itatani, J. Levesque, D. Zeidler, H. Niikura, H. Pépin, J. C. Kieffer, P. B. Corkum, and D. M. Villeneuve, Nature (London) 432, 867 (2004).

[6] D. Pavičić, K. F. Lee, D. M. Rayner, P. B. Corkum, and D. M. Villeneuve, Phys. Rev. Lett. 98, 243001 (2007).

[7] I. Thomann, R. Lock, V. Sharma, E. Gagnon, S. T. Pratt, H. C. Kapteyn, M. M. Murnane, and W. Li, J. Phys. Chem. A 112, 9382 (2008)

[8] M. Meckel, D. Comtois, D. Zeidler, A. Staudte, D. Pavičić, H. C. Bandulet, H. Pépin, J. C. Kieffer, R. Dörner, D. M. Villeneuve, and P. B. Corkum, Science 320, 1478 (2008).

[9] B. K. McFarland, J. P. Farrell, P. H. Bucksbaum, and M. Gühr, Science 322, 1232 (2008).

[10] M. Lein, J. Phys. B 40, R135 (2007).

[11] J. Muth-Böhm, A. Becker, and F. H. M. Faisal, Phys. Rev. Lett. 85, 2280 (2000).

[12] X. M. Tong, Z. X. Zhao, and C. D. Lin, Phys. Rev. A 66, 033402 (2002).

[13] C. B. Madsen and L. B. Madsen, Phys. Rev. A 74, 023403 (2006).

[14] Z. Chen, T. Morishita, A. T. Le, and C. D. Lin, Phys. Rev. A 76, 043402 (2007).

[15] S. Odžak and D. B. Milošević, Phys. Rev. A 79, 023414 (2009).

[16] S. Patchkovskii, Z. Zhao, T. Brabec, and D. M. Villeneuve, Phys. Rev. Lett. 97, 123003 (2006).

[17] R. Santra and A. Gordon, Phys. Rev. Lett. 96, 073906 (2006).

[18] D. A. Telnov and Shih-I Chu, Phys. Rev. A 79, 041401(R) (2009).

[19] S. I. Chu, J. Chem. Phys. 123, 062207 (2005).

[20] X. Chu and Shih-I Chu, Phys. Rev. A 70, 061402(R) (2004).

[21] S. K. Son and Shih-I Chu, Phys. Rev. A 80, 011403(R) (2009).

[22] Time-Dependent Density Functional Theory, edited by M. A. L. Marques, C. A. Ullrich, F. Nogueira, A. Rubio, K. Burke, and E. K. U. Gross (Springer, Berlin, 2006).

[23] P. R. T. Schipper, O. V. Gritsenko, S. J. A. van Gisbergen, and E. J. Baerends, J. Chem. Phys. 112, 1344 (2000).

[24] J. Heslar, J. Carrera, D. Telnov, and S. I. Chu, Int. J. Quantum
Chem. 107, 3159 (2007).

[25] D. A. Telnov and Shih-I Chu, Phys. Rev. A 76, 043412 (2007).

[26] D. A. Telnov and Shih-I Chu, Phys. Rev. A 71, 013408 (2005).

[27] X. Chu and Shih-I Chu, Phys. Rev. A 63, 023411 (2001).

[28] X. Chu and Shih-I Chu, Phys. Rev. A 64, 063404 (2001).

[29] Handbook of Mathematical Functions, edited by M. Abramowitz and I. Stegun (Dover, New York, 1965).

[30] L. Tao, C. W. McCurdy, and T. N. Rescigno, Phys. Rev. A 79, 012719 (2009).

[31] C. C. Marston and G. G. Balint-Kurti, J. Chem. Phys. 91, 3571 (1989).

[32] K. P. Huber and G. Herzberg, Molecular Spectra and Molecular Structure. IV. Constants of Diatomic Molecules (Van Nostrand Reinhold Co., New York, 1979).

[33] A. Lofthus and P. H. Krupenie, J. Phys. Chem. Ref. Data 6, 113 (1977).

[34] A. B. Cornford, D. C. Frost, C. A. McDowell, J. L. Ragle, and I. A. Stenhouse, J. Chem. Phys. 54, 2651 (1971).

[35] K. Siegbahn, C. Nordling, G. Johansson, J. Hedman, P. F. Hedén, K. Hamrin, U. Gelius, T. Bergmark, L. O. Werme, R. Manne, and Y. Baer, ESCA Applied to Free Molecules (North Holland, Amsterdam, 1969).

[36] V. I. Usachenko, P. E. Pyak, and V. V. Kim, Phys. Rev. A 79, 023415 (2009).

[37] L. D. Landau and E. M. Lifshitz, The Classical Theory of Fields (Pergamon Press, Oxford, New York, 1975).

[38] P. B. Corkum, Phys. Rev. Lett. 71, 1994 (1993).

[39] J. A. R. Samson and W. C. Stolte, J. Electron Spectrosc. Relat. Phenom. 123, 265 (2002).

[40] W. F. Chan, G. Cooper, X. Guo, G. R. Burton, and C. E. Brion, Phys. Rev. A 46, 149 (1992).

[41] S. Minemoto, T. Umegaki, Y. Oguchi, T. Morishita, A. T. Le, S. Watanabe, and H. Sakai, Phys. Rev. A 78, 061402(R) (2008).

[42] A. T. Le, T. Morishita, and C. D. Lin, Phys. Rev. A 78, 023814 (2008).

[43] M. Lein, N. Hay, R. Velotta, J. P. Marangos, and P. L. Knight, Phys. Rev. A 66, 023805 (2002).

[44] X. Zhou, R. Lock, W. Li, N. Wagner, M. M. Murnane, and H. C. Kapteyn, Phys. Rev. Lett. 100, 073902 (2008).

[45] B. Zimmermann, M. Lein, and J. M. Rost, Phys. Rev. A 71, 033401 (2005). 